\title{
Combined Optical and Electronic Readout for Event Reconstruction in a GEM-Based TPC
}

\author{
Florian M. Brunbauer ${ }^{\circledR}$, Francisco Garcia, Tero Korkalainen, Alois Lugstein, Michael Lupberger ${ }^{\circledR}$, \\ Eraldo Oliveri, Dorothea Pfeiffer, Leszek Ropelewski, Patrik Thuiner, and Markus Schinnerl
}

\begin{abstract}
Optically read out time projection chambers (TPCs) based on gaseous electron multipliers (GEMs) combine 3-D event reconstruction capabilities with high spatial resolution and charge amplification factors. The approach of reconstructing particle tracks from 2-D projections obtained with imaging sensors and depth information from photomultiplier tubes is limited to specific cases such as straight particle trajectories. A combination of optical and electronic readout realized by a semitransparent anode placed between a triple-GEM stack and a camera in an optically read out TPC has been realized and used to reconstruct more complex particle tracks. High spatial resolution 2-D projections combined with a low number of charge readout channels enable accurate 3-D event topology reconstruction. Straight alpha tracks as well as more complex cosmic events have been reconstructed with the presented readout concept. Relative depth information from electronically read out charge signals has been combined with drift time information between primary and secondary scintillation pulses to absolute alpha track reconstructions.
\end{abstract}

Index Terms-Event building, gaseous electron multiplier (GEM) detectors, micropattern gas chambers, optical signal detection, particle detectors, position sensitive detectors, proportional gas scintillation detectors, radiation detectors, readout systems, scintillation detectors, signal reconstruction, time projection chambers (TPCs).

\section{INTRODUCTION}

T IME projection chambers (TPCs) [1] are a powerful tool allowing 3-D particle track reconstruction from 2-D projections and associated depth information. Due to their applicability for a variety of different particles, energies, and track topologies, TPCs are at the core of numerous running experiments such as the ALICE experiment [2] at the LHC at CERN and the STAR experiment [3] at the RHIC at BNL or previous experiments such as the ALEPH [4] and DELPHI [5]

Manuscript received November 13, 2017; revised January 24, 2018; accepted January 29, 2018. Date of publication February 1, 2018; date of current version March 14, 2018.

F. M. Brunbauer is with the Institute of Atomic and Subatomic Physics, Technische Universität Wien, 1040 Vienna, Austria, and also with the EP Division, CERN, 1211 Geneva, Switzerland (e-mail: florian.brunbauer@cern.ch).

F. Garcia is with the Helsinki Institute of Physics, University of Helsinki, 00014 Helsinki, Finland.

T. Korkalainen was with the EP Division, CERN, 1211 Geneva, Switzerland. $\mathrm{He}$ is now with the School of Electrical Engineering, Aalto University, 00076 Espoo, Finland.

A. Lugstein and M. Schinnerl are with the Institute for Solid State Electronics, Technische Universität Wien, 1040 Vienna, Austria.

M. Lupberger, E. Oliveri, D. Pfeiffer, L. Ropelewski, and P. Thuiner are with the EP Division, CERN, 1211 Geneva, Switzerland.

Color versions of one or more of the figures in this paper are available online at http://ieeexplore.ieee.org.

Digital Object Identifier 10.1109/TNS.2018.2800775 experiments at the LEP at CERN. Future experiments in a variety of fields are also centered around TPCs such as the NEXT experiment for neutrinoless double-beta decay search [6], liquid Xe-based TPCs searching for nuclear recoils resulting from scattering dark matter particles [7] or the DMTPC project focused on directional dark matter search [8] or for the tracking of heavy ions at the Super-FRS [9] at FAIR, to name but a few.

Micropattern gaseous detector (MPGD) technologies such as gaseous electron multipliers (GEMs) [10] offer good spatial resolution combined with sensitivity to a wide range of radiation due to the high achievable charge gain factors. These properties in addition to economic scalability and the possibility to realize low-material-budget detectors owing to the usage of gas as active medium make them well suited for the readout of track projections on the endcaps of TPCs. A number of TPCs with GEM-based endcap readout schemes have been proposed, developed, or operated [2], [11]-[13].

Taking advantage of secondary scintillation light emitted during electron avalanche multiplication, GEMs operated in suitable gas mixtures can be optically read out by imaging sensors [14], [15]. The high pixel count of state-of-the-art chargecoupled device (CCD) or CMOS imaging sensors permits high spatial resolution optical readout. The 2-D images of detected tracks can be obtained from cameras without the need for extensive reconstruction algorithms. Combined with the depth of interaction information such as from fast photon detectors like photomultiplier tubes (PMTs), 3-D track reconstruction in optically read out TPCs can be realized [16]. The width of the secondary scintillation pulse recorded by the PMT can be used to extract relative depth information between the beginning and end points of particle tracks. In addition to PMT waveforms, some sensitivity to relative $Z$-information, i.e., depth information along the axis of the active volume of a TPC, can also be achieved by reading out electronic anode signals with a fast amplifier as used for track reconstruction in the DMTPC project [17]. The absolute $Z$-position, i.e., the distance from the endcap of the active volume of a TPC, of a particle track can be computed by multiplying the difference between the time $t_{0}$ of the crossing of the active detection volume signaling the beginning of an event and the time of the detection of an event on the endcap of a TPC with the electron drift velocity. For particles that can traverse multiple detector layers, $t_{0}$ can be determined by external scintillators or other detector technologies providing a timestamp for the entry of a particle into the TPC. Alternatively, drift time information 


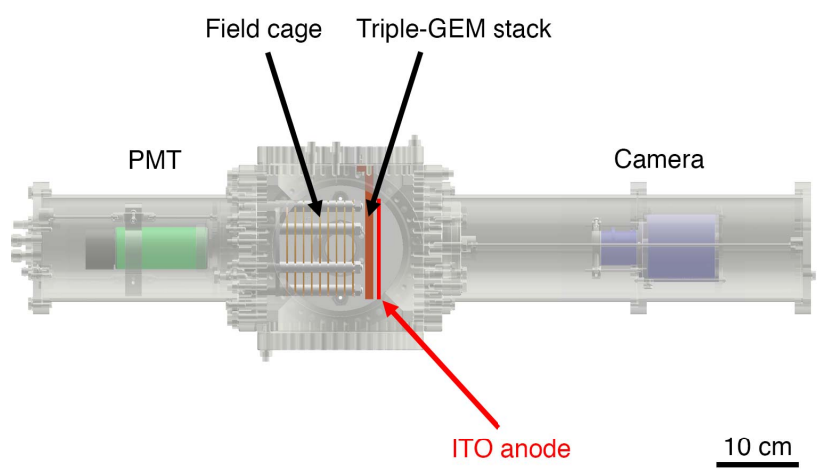

(a)

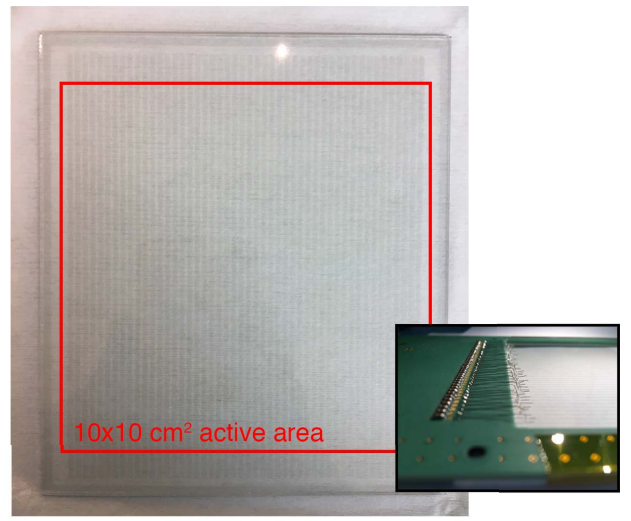

(b)

Fig. 1. (a) Schematic of GEM-based TPC read out by PMT and camera with ITO anode below triple-GEM. The transparent anode is placed between the triple-GEM and the camera used for optical readout. The active volume is formed by a cylindrical field cage. The detector components are integrated in an ultra-high vacuum-grade vessel filled with $\mathrm{Ar} / \mathrm{CF}_{4}(80 \% / 20 \%)$ at an absolute pressure of 1 bar. (b) ITO strip anode with 48 strips covering the $10 \times 10 \mathrm{~cm} 2$ active area shown by a red frame. Inset: Contact wires between adapter PCB and individual ITO strips.

can also be extracted from the time between primary and secondary scintillation pulses in PMT waveforms if particles produce sufficient primary scintillation light to be detectable. The orientation of particle tracks may be determined from matching energy loss profiles in PMT waveforms and optically read out images and identifying the location of the Bragg peak for particles with nonuniform energy loss [18]. Optically read out TPCs have been employed for studies in nuclear astrophysics with gamma-ray beams [19], [20], proton spectroscopy [21], and studies of two-proton decays [22], among other applications.

The concept of 3-D track reconstruction from a combination of 2-D projections from imaging sensors and depth information from PMT waveforms is limited to straight tracks. While this may be acceptable for tracks such as alpha particles since their trajectories are straight, more complex track topologies or even curved particle trajectories may not be unambiguously reconstructed by this approach due to the lack of intermediate depth information between the start and end points of a track.

To extend the reconstruction capabilities of optically read out TPCs, we present a readout approach based on a combination of optical and electronic readout. The 2-D projections are obtained by recording secondary scintillation light emitted by a triple-GEM stack in a TPC setup with a camera, while depth information is extracted from differences in the arrival time of charge signals on a segmented transparent anode. Simultaneous readout of charge signals from an anode below the triple-GEM stack and secondary scintillation through the anode was made possible by using a patterned indium tin oxide (ITO) film as anode. ITO can be deposited by evaporation or sputtering techniques, and ITO films are widely used as optically transparent shielding layers against electromagnetic interference. ITO-coated glass substrates have previously been used in optically read out GEM-based detectors to record charge spectra without spatial information [23].

The presented device was used to obtain 3-D visualizations of alpha particle tracks as well as curved and more complex cosmic events. Although the absolute $Z$-position of particle tracks cannot be determined only from arrival time information of charge signals, the presented readout concept can provide relative depth information and accurate topological visualizations of complex particle tracks. The absolute depth of particle tracks can be determined if the time $t_{0}$ of the occurrence of an event in the active volume of the TPC can be determined.

\section{EXPERIMENT}

An optically read out TPC with a triple-GEM stack with an active area of $10 \times 10 \mathrm{~cm}^{2}$ was implemented in a vacuum-grade vessel. Transfer gaps with a thickness of $2 \mathrm{~mm}$ between individual GEM foils were chosen, and GEM electrodes were individually connected to a resistor-based voltage divider outside of the detector vessel through safe high voltage feedthroughs. The active drift region of the TPC was formed by a cylindrical field cage with a length of $10 \mathrm{~cm}$ and a diameter of $10 \mathrm{~cm}$ made of ring-shaped $\mathrm{Cu}$ electrodes at a spacing of $1 \mathrm{~cm}$. The individual electrodes of the field cage were biased via resistors in the detector vessel forming a resistive voltage divider. A semitransparent stainless steel mesh was used as cathode. Borosilicate viewports with a clear view diameter of $63 \mathrm{~mm}$ on opposite sides of the detector vessel permitted the readout of scintillation light from the detector. A 2-in PMT (Hamamatsu R375) was placed outside of the vessel facing the drift volume through the cathode mesh. An electron multiplying CCD (EMCCD) camera (Hamamatsu ImagEM $\mathrm{X} 2$ ) was placed on the opposite side outside of the chamber facing the bottom of the triple-GEM stack. A segmented ITO anode was placed below the triple-GEM stack with an induction gap with a thickness of $4 \mathrm{~mm}$. The detector and readout components in the TPC setup are schematically shown in Fig. 1(a).

The ITO anode was manufactured from a glass plate with a thickness of $1.1 \mathrm{~mm}$ coated with a thin ITO layer. Initially, a $5 \times 5$ pad design was implemented in an ITO layer with a thickness of $25 \mathrm{~nm}$. The high series resistance due to the high sheet resistivity of $100 \Omega^{-2}$ of the thin ITO layer and the low granularity of the $2 \times 2 \mathrm{~cm}^{2}$ pads in this design proved to be 
unsuitable for accurate track reconstruction. Therefore, an ITO strip anode based on a thicker ITO layer was developed. A $11 \times 12 \mathrm{~cm}^{2}$ glass plate coated with an ITO layer with a thickness of $450 \mathrm{~nm}$ corresponding to a sheet resistivity of $4 \Omega^{-2}$ was used as base material to manufacture an ITO strip anode with 48 strips with a strip width of $1.5 \mathrm{~mm}$ at a pitch of $2 \mathrm{~mm}$ covering the $10 \times 10 \mathrm{~cm}^{2}$ active area as shown in Fig. 1(b). A photoresist layer was applied to the ITO-coated glass plate by spin coating. Subsequently, the anode strip pattern was exposed by direct laser lithography with a resolution of about $2.5 \mu \mathrm{m}$. After the wet-chemical development of the photoresist layer, ITO in exposed regions was removed by etching in a bath of hydrochloric acid $(\mathrm{HCl})$ with a concentration of $32 \%$ for $17 \mathrm{~min}$. The photoresist layer was then removed with acetone, and the ITO strip anode was cleaned with dry nitrogen. Next, the ITO strip anode was glued onto a ceramic frame and fixed to an adapter printed circuit board (PCB) before being placed in the detector vessel below the triple-GEM stack.

The ITO anode was electronically read out with an APV25 readout application-specified integrated circuit (ASIC) [24] on a hybrid board connected to the RD51 scalable readout system (SRS) [25]. The APV25 ASIC features 128 readout channels and can record signals in 27 time bins of $25 \mathrm{~ns}$ each resulting in a total width of the acquisition window of $675 \mathrm{~ns}$. For an electron drift velocity of $8 \mathrm{~cm} / \mu \mathrm{s}$, this corresponds to a maximum recordable drift distance of $5.4 \mathrm{~cm}$. The adapter PCB was used to connect the individual strips of the ITO strip anode to the readout channels of the APV25 hybrid board. The electrical contact between ITO strips and the adapter PCB was made by individual metallic wires bent into place and glued to the ITO strips with Ag-loaded adhesive. The APV25 hybrid board was placed in the detector vessel and connected to the SRS by high-definition multimedia interface cables and a vacuum-compatible multipin feedthrough.

The APV25 ASIC requires an external signal to trigger data acquisition. This trigger signal was generated with nuclear instrumentation module-based electronics from a PMT signal of the secondary scintillation produced during avalanche multiplication in the GEM stack or from an electronic signal from the bottom electrode of the last GEM in the triple-GEM stack. Electronic modules were used to handle the trigger logic of the system in order to only accept events while all components of the readout system were ready for data acquisition. The EMCCD camera was operated in the bulb triggering mode with low voltage complementary metal oxide semiconductor signals used to define the exposure window. The exposure of the camera was started as soon as the system was ready for the next event, and the shutter was closed as soon as a new event was registered. Subsequently, the image from the camera was read out and stored to be available for offline event reconstruction.

Prior to gas filling, the detector vessel was pumped by a turbomolecular pump backed by a rotary vane pump to clean the chamber and minimize outgassing from detector components in contact with the gas. The detector vessel was filled with a $\mathrm{Ar} / \mathrm{CF}_{4}(80 \% / 20 \%$ by volume) gas mixture at 1 bar and operated in the open gas flow mode. This gas

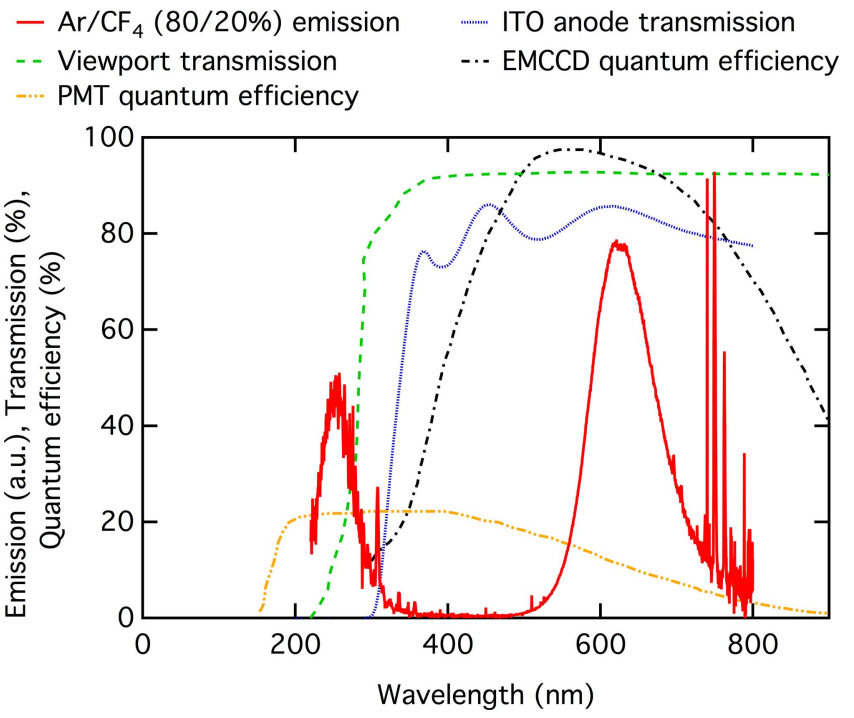

Fig. 2. Emission spectrum of the secondary scintillation of $\mathrm{Ar} / \mathrm{CF}_{4}$ $(80 \% / 20 \%)$ (red solid curve) features broad emission bands in visible and UV wavelength ranges and some sharp emission peaks in the near infrared response. The transmission of the ITO strip anode (blue dotted curve) exhibits some undulation and more than $80 \%$ transmission in the visible range. The borosilicate viewport is highly transparent over a wide range of wavelengths (green dashed curve). The quantum efficiency of the EMCCD camera peaks in the visible wavelength range (black dashed-dotted curve). The quantum efficiency of the PMT (orange dashed double dotted curve) is about $11 \%$ at $630 \mathrm{~nm}$.

mixture offered a high secondary scintillation light yield of up to 0.3 photons per secondary electron and features a scintillation light emission spectrum compatible with the employed imaging sensor. The emission spectrum of the gas mixture used in the presented TPC is shown in Fig. 2 along with the quantum efficiency curve of the EMCCD camera. The pronounced emission band in the visible wavelength regime peaks at $630 \mathrm{~nm}$ and matches the quantum efficiency of the EMCCD well. The borosilicate viewport in the optical path of the recorded secondary scintillation light has high transmission in this wavelength range. The ITO strip anode between the triple-GEM and the viewport and the EMCCD camera also features an optical transmission of more than $80 \%$ at $630 \mathrm{~nm}$ as shown in Fig. 2. The ITO layer thickness of $450 \mathrm{~nm}$ results in some undulation of the wavelength-dependent transmission of the anode, but the achieved transmission was sufficient for optical readout.

With an electron multiplier gain of up to $1200 \times$, the employed EMCCD camera was well suited for optical readout even in low-light conditions. Featuring pixels with a size of $16 \times 16 \mu \mathrm{m}^{2}$ and a resolution of $512 \times 512$ pixels, the camera could provide $2-\mathrm{D}$ images with sufficient spatial resolution. The low readout noise of 1 electron max. (rms) and a dark current of 0.0005 electrons/pixel/s at a sensor temperature of $-80{ }^{\circ} \mathrm{C}$ permitted high signal-to-noise ratios in optically obtained images despite varying exposure times due to the employed bulb mode triggering scheme.

For highly ionizing alpha particles, a GEM gain of $4 \times 10^{3}$ was sufficient while the triple-GEM stack was operated with a higher gain of $1.5 \times 10^{4}$ for cosmic events to achieve sufficient secondary scintillation light emission. The gain of the PMT used to obtain trigger signals from secondary scintillation 


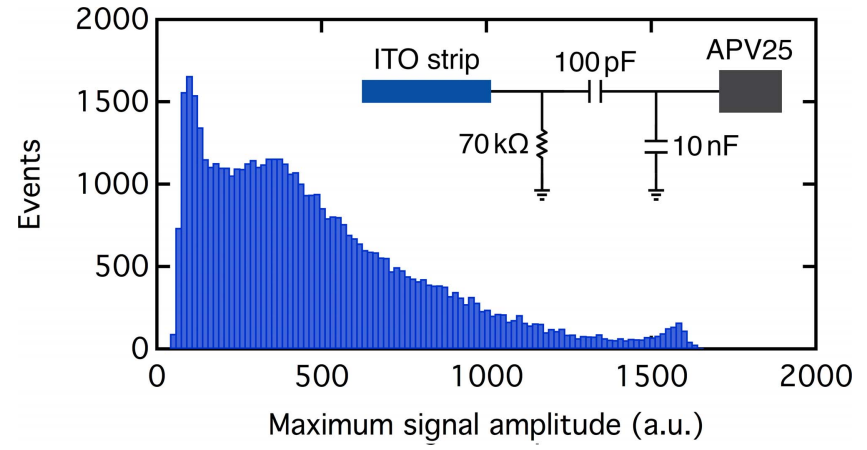

Fig. 3. Distribution of maximum ITO strip signal amplitudes for 5000 alpha events with $100 \times$ attenuation factor. Inset: Capacitive voltage divider between ITO strips and APV readout channels.

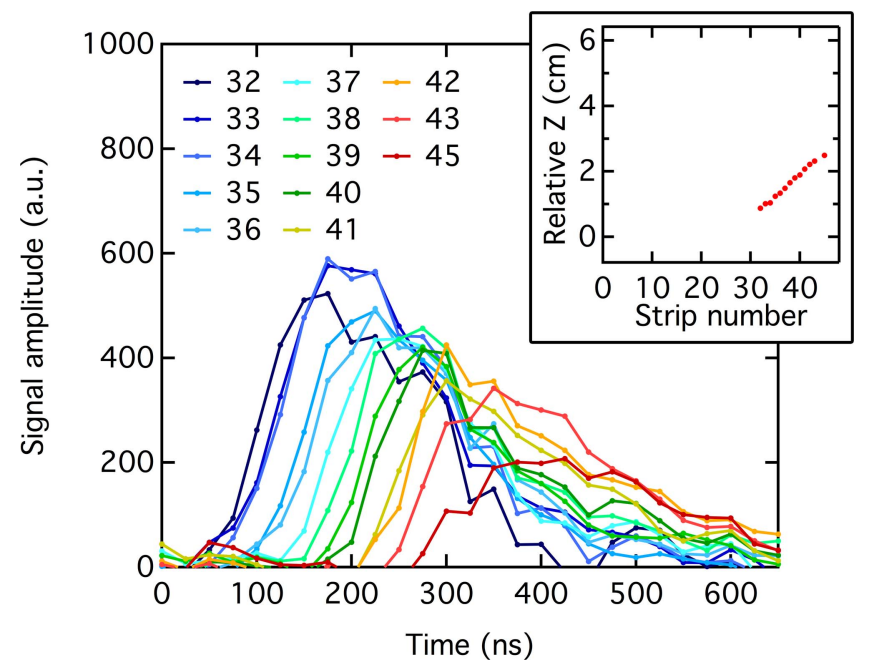

Fig. 4. Electronic signals on ITO strips with numbers of 32-45 recorded by APV25 ASIC. Inset: Relative depth information obtained from differences in arrival time of electronic signals.

pulses was adjusted from $2 \times 10^{5}$ to $2 \times 10^{6}$ depending on the type of events recorded. The EMCCD camera was operated with electron multiplier gains of $1000 \times$ or $1200 \times$ for alpha particles or cosmic events, respectively. A drift field strength of $300 \mathrm{~V} / \mathrm{cm}$ was used, and a low induction field strength of about $650 \mathrm{~V} / \mathrm{cm}$ was chosen to decrease the amplitude of the signals on the anode.

While optical readout demands high-GEM gain factors to achieve sufficient secondary scintillation light emission, the limited dynamic range of the APV25 ASIC might result in signal saturation. To mitigate this problem, capacitive voltage dividers integrated in the adapter PCB with an attenuation factor of $100 \times$ as shown in the inset of Fig. 3 were used between the individual strips of the ITO anode and the readout channels of the APV25 ASIC. This allowed the recording of high signal-to-noise ratio images with minimal saturation in the recorded electronic signals as shown by the distribution of maximum electronic signal amplitudes in Fig. 3.

\section{RESUlTS AND DISCUSSION}

The track reconstruction capabilities of the TPC were investigated with a gaseous source of alpha particles with

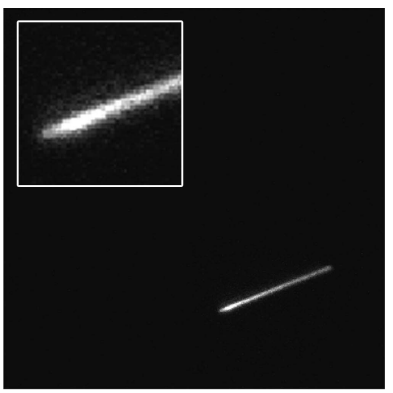

(a)

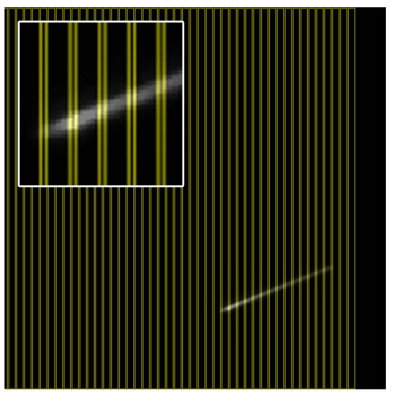

(c)

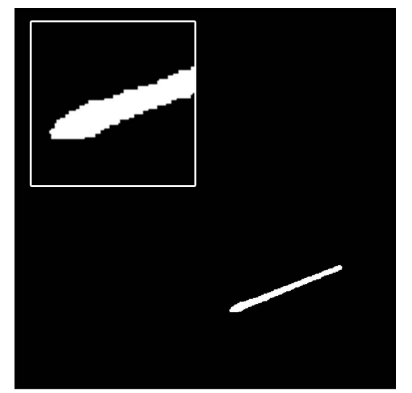

(b)

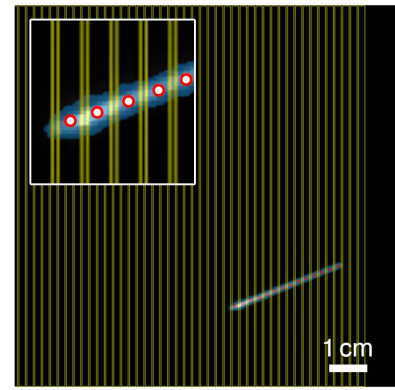

(d)
Fig. 5. (a) Optically read out images of particle tracks are processed by subtracting a background image and applying median filtering. (b) Fixed threshold is used to obtain a binary image. (c) Strip pattern of the ITO anode is shown as overlay (yellow). (d) Bright pixels are identified (blue), and the mean values of the $X$ and $Y$ coordinates of all bright pixels within a strip are used to determine the 2-D hit position in a strip (white dots with red circles).

an energy of $6.4 \mathrm{MeV}$ from the decay of ${ }^{220} \mathrm{Rn}$ flushed into the detector vessel as well as with cosmic events. Electronic signals of an exemplary alpha track are shown in Fig. 4. In this event, charge signals on more than ten adjacent strips were recorded. The arrival time of the signal on each anode strip was determined by a constant fraction discrimination algorithm with linear interpolation between data points at $30 \%$ of the maximum signal amplitude. The extracted arrival times were then multiplied by the known drift velocity of $8 \mathrm{~cm} / \mu$ s of electrons in a $300 \mathrm{~V} / \mathrm{cm}$ drift field in the employed gas mixture to obtain relative $Z$-depth information for the hit strips as shown in the inset of Fig. 4. The width of the time bins of the APV25 ASIC limits the achievable $Z$-depth resolution. For an electron drift velocity of $8 \mathrm{~cm} / \mu \mathrm{s}$, the time-bin width of the APV25 ASIC of $25 \mathrm{~ns}$ corresponds to a $Z$-depth range of $2 \mathrm{~mm}$. Although the $Z$-resolution may be improved by interpolation between data points on the rising edge of the electronic signals, the coarse sampling by the APV25 remains a factor limiting $Z$-resolution.

Optically read out images as shown in Fig. 5(a) were used to obtain 2-D projections of particle tracks. In a first image processing step, an averaged background image was subtracted from raw images. Subsequently, outliers such as hot pixels were removed by a $4 \times 4$ median filter, and the resulting images were converted to binary images with a fixed threshold of several standard deviations above the noise level as shown in Fig. 5(b). The pattern of the ITO strip anode was then overlaid onto the image [Fig. 5(c)], and bright pixels within each anode strip were identified. As only a single value 

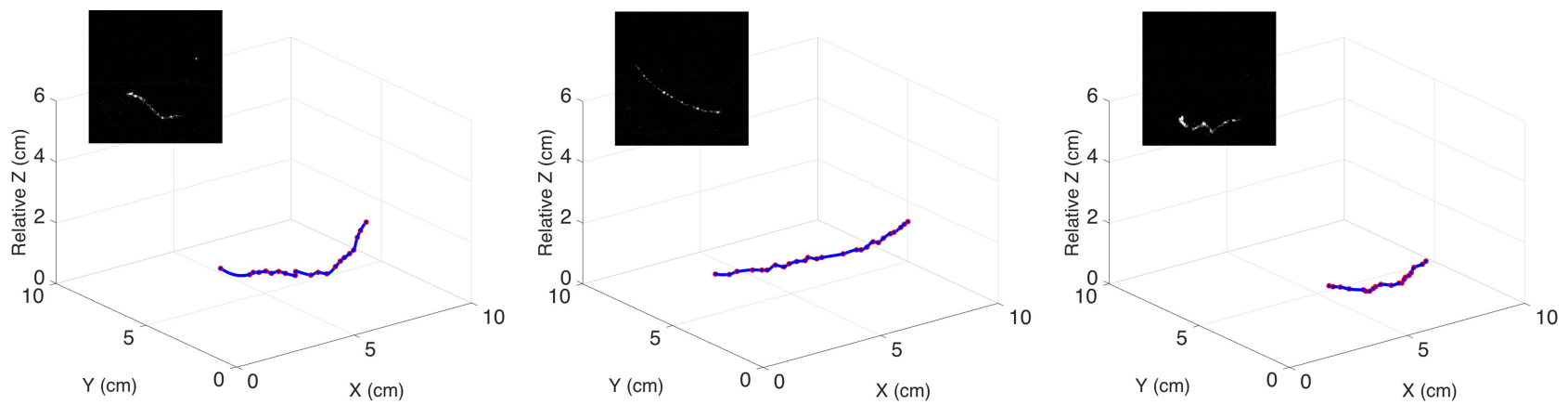

Fig. 6. Cosmic events reconstructed by the combination of optical and electronic readout with ITO strip anode. Inset: Corresponding 2-D projections of the events. The relative depth information permits a reconstruction of curved and more complex particle trajectories. Red dots are reconstructed 3-D track points. Blue curves display tracks obtained by cubic spline interpolation between reconstructed 3-D points.

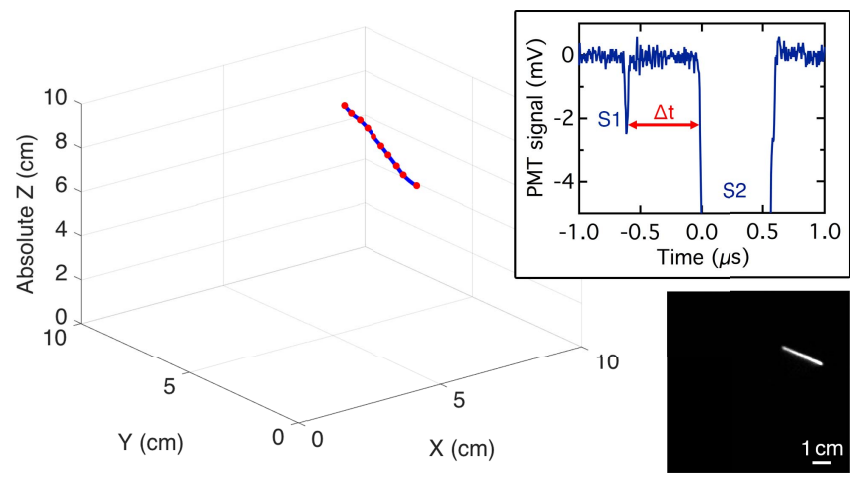

Fig. 7. Alpha particle track reconstructed by combining 2-D optical image (shown in lower right corner) with relative depth information from ITO strip anode and absolute $Z$-information from time difference $\Delta t$ between primary (S1) and secondary (S2) scintillation pulses in PMT waveform shown in the inset.

for the $Z$-coordinate within a certain strip was provided by the electronic readout, this value needed to be assigned to a certain 2-D position. The mean values of the $X$ and $Y$ coordinates of all bright pixels within a certain anode strip region were calculated and used as the hit position within a certain anode strip, as shown in Fig. 5(d).

The combination of 2-D hit locations from optical images with relative $Z$-information resulted in a number of 3-D reconstructed points along the detected particle tracks. Track visualizations were subsequently obtained by cubic spline interpolation between the reconstructed 3-D points. Examples of reconstructed cosmic events are shown in Fig. 6 along with the corresponding optical images.

While track reconstruction worked well for events in which several anode strips were hit, tracks almost parallel to the anode strips could not be accurately reconstructed. The angular resolution of the presented device could not be determined as the alpha particles had a random distribution of origins and orientations due to the use of a gaseous radiation source.

To determine the absolute position of particle tracks in the TPC, the time $t_{0}$ of the passage of a track is required. For highly ionizing particles such as alpha tracks, primary scintillation might be sufficiently strong to be recorded by highly sensitive photon detectors such as PMTs. The absolute depth of interaction of alpha particle tracks in the presented optically read out TPC was determined by this approach in addition to the 2-D projections from optical images and the relative depth information from the arrival times of electronically read out charge signals. The time difference $\Delta t$ between the primary scintillation pulse and the leading edge of the secondary scintillation pulse as shown in Fig. 7 corresponds to the drift time of the primary electrons produced closest to the triple-GEM stack. In the shown example, a drift time of $\Delta t=612 \mathrm{~ns}$ corresponds to a distance of $4.89 \mathrm{~cm}$ for an electron drift velocity of $8 \mathrm{~cm} / \mu \mathrm{s}$. This distance is used as the absolute $Z$-coordinate of the track point closest to the triple-GEM stack. The relative $Z$-information from the ITO anode is used to determine $Z$-coordinates along the rest of the track. This combination of 2-D images, electronically read out charge signals, and PMT waveforms enables an absolute 3-D particle track reconstruction.

\section{CONCLUSION}

The reconstruction capabilities of optically read out TPCs can be extended to curved and more complex particle trajectories by combining optically read out images with arrival time information of charge signals obtained by electronic readout. Optically transparent and electrically conductive anodes for MPGDs can be manufactured from ITO-coated substrates and patterned by standard photolithographic techniques. The optical transparency of ITO films on glass substrates is well suited for the optical readout of scintillation light emitted in $\mathrm{Ar} / \mathrm{CF}_{4}$ gas mixtures.

Electronic signal attenuation or readout ASICs with a wider dynamic range may be used to minimize saturation in electronically read out signals despite the high charge gain factors required for optical readout. The width of time bins in the APV25 ASIC limits the achievable Z-resolution in the TPC. The use of a fast multichannel analog-to-digital converter or a readout ASIC with better time sampling capabilities may be used to achieve better $Z$-resolution.

Relative $Z$-information can be obtained from electronically read out signals without the need for fast photon detectors. In addition, absolute $Z$-information extracted from the time between primary and secondary scintillation pulses in PMT waveforms can be used to determine the absolute position of highly ionizing particle tracks with sufficient primary scintillation light emission to be recorded. 
The 1-D strip anode might not give accurate depictions of particle tracks, which are almost parallel to the anode strips. A 2-D strip anode, a pixelated anode, or a combination of strips and wires might be used to obtain some 2-D spatial information associated with the arrival times of electronic signals. Even in this case, the number of required electronic readout channels can remain low as 2-D projections with high granularity are obtained by optical readout.

The 3-D event reconstruction capabilities of a GEM-based TPC employing a combination of optical and electronic readout have been demonstrated for alpha particles and cosmic events. The presented readout concept extends the reconstruction capabilities of optically read out TPCs and might be used to obtain topological information in applications in the search for rare events such as directional dark matter search or searches for neutrinoless double-beta decay.

\section{ACKNOWLEDGMENT}

The authors would like to thank C. Bault and M. van Stenis from CERN, Geneva, Switzerland, for their support with the design, manufacturing, and assembly of the experimental setup. They would like to thank H. Müller, F. Sauli, and T. Schneider from CERN, for their support and scientific input to the presented work. They would also like to thank the Center for Micro- and Nanostructures (ZMNS), Vienna, Austria, for providing clean room facilities.

\section{REFERENCES}

[1] D. R. Nygren, "The time projection chamber: A new 4 pi detector for charged particles," in Proc. eConf, 1974, pp. 58-78.

[2] J. Alme et al., "The ALICE TPC, a large 3-dimensional tracking device with fast readout for ultra-high multiplicity events," Nucl. Instrum. Methods Phys. Res. A, Accel. Spectrom. Detect. Assoc. Equip., vol. 622, no. 1, pp. 316-367, 2010.

[3] K. H. Ackermann et al., "The forward time projection chamber in STAR," Nucl. Instrum. Methods Phys. Res. A, Accel. Spectrom. Detect. Assoc. Equip., vol. 499, nos. 2-3, pp. 713-719, 2003.

[4] W. B. Atwood et al., "Performance of the ALEPH Time Projection Chamber," Nucl. Instrum. Methods Phys. Res. A, Accel. Spectrom. Detect. Assoc. Equip., vol. 306, no. 3, pp. 446-458, 1991

[5] C. Brand et al., "The DELPHI time projection chamber," Nucl. Instrum. Methods Phys. Res. A, Accel. Spectrom. Detect. Assoc. Equip., vol. 283, no. 3, pp. 567-572, 1989.

[6] D. Lorca, J. Martín-Albo, and F. Monrabal, "The NEXT experiment: A high pressure xenon gas TPC for neutrinoless double beta decay searches," Nucl. Instrum. Methods Phys. Res. A, Accel. Spectrom. Detect. Assoc. Equip., vol. 718, pp. 387-390, Aug. 2013.

[7] E. Aprile et al., "The XENON100 dark matter experiment," Astropart. Phys., vol. 35, no. 9, pp. 573-590, 2012.
[8] G. Sciolla, "The DMTPC project," J. Phys., Conf. Ser., vol. 179, no. 1, p. $12009,2009$.

[9] F. García et al., "Twin GEM-TPC prototype (HGB4) beam test at GSI and jyväskylä-A development for the super-FRS at FAIR," in Proc. IEEE Nucl. Sci. Symp., Med. Imag. Conf. Room-Temperature Semiconductor Detector Workshop (NSS/MIC/RTSD), vol. 4. Oct. 2016 , pp. $1-5$.

[10] F. Sauli, "GEM: A new concept for electron amplification in gas detectors," Nucl. Instrum. Methods Phys. Res. A, Accel. Spectrom. Detect. Assoc. Equip., vol. 386, nos. 2-3, pp. 531-534, Feb. 1997.

[11] L. Fabbietti et al., "The PANDA GEM-based TPC prototype," Nucl. Instrum. Methods Phys. Res. A, Accel. Spectrom. Detect. Assoc. Equip., vol. 628, no. 1, pp. 204-208, 2011.

[12] N. S. Phan, R. J. Lauer, E. R. Lee, D. Loomba, J. A. J. Matthews, and E. H. Miller, "GEM-based TPC with CCD imaging for directional dark matter detection," Astroparticle Phys., vol. 84, pp. 82-96, Nov. 2016.

[13] K. Ikematsu, "Development of large-aperture GEMs as a gating device of the ILC-TPC for blocking positive ion feedback," in Proc. IEEE Nucl. Sci. Symp. Med. Imag. Conf. (NSS/MIC), Nov. 2014, pp. 1-4.

[14] F. A. F. Fraga, S. T. G. Fetal, L. M. S. Margato, I. Ivaniouchenkov, R. F. Marques, and A. J. P. L. Policarpo, "Imaging detectors based on the gas electron multiplier scintillation light," in Proc. IEEE Nucl. Sci. Symp. Conf. Rec., vol. 2. Oct. 1999, pp. 829-833.

[15] F. A. F. Fraga, L. M. S. Margato, S. T. G. Fetal, M. M. F. R. Fraga, R. F. Marques, and A. J. P. L. Policarpo, "Luminescence and imaging with gas electron multipliers," Nucl. Instrum. Methods Phys. Res. A, Accel. Spectrom. Detect. Assoc. Equip., vol. 513, nos. 1-2, pp. 379-387, 2003.

[16] P. Fonte, A. Breskin, G. Charpak, W. Dominik, and F. Sauli, "Beam test of an imaging high-density projection chamber," Nucl. Instrum. Methods Phys. Res. A, Accel. Spectrom. Detect. Assoc. Equip., vol. 283, no. 3, pp. 658-664, 1989.

[17] C. Deaconu et al., "Track reconstruction progress from the DMTPC directional dark matter experiment," Phys. Procedia, vol. 61, pp. 39-44, Jan. 2015.

[18] F. M. Brunbauer et al., "Live event reconstruction in an optically read out GEM-based TPC," Nucl. Instrum. Methods Phys. Res. A, Accel. Spectrom. Detect. Assoc. Equip., vol. 886, pp. 24-29, Apr. 2018.

[19] M. Gai et al., "An optical readout TPC (O-TPC) for studies in nuclear astrophysics with gamma-ray beams at $\mathrm{HI}_{y} \mathrm{~S}^{1}$, "J. Instrum., vol. 5, no. 12, p. P12004, 2010.

[20] W. R. Zimmerman et al., "Unambiguous identification of the second $2^{+}$State in ${ }^{12} \mathrm{C}$ and the structure of the hoyle state," Phys. Rev. Lett., vol. 110, no. 15, p. 152502, 2013.

[21] M. Pomorski et al., "Proton spectroscopy of ${ }^{48} \mathrm{Ni},{ }^{46} \mathrm{Fe}$, and ${ }^{44} \mathrm{Cr}$," Phys. Rev. C, Nucl. Phys., vol. 90, no. 1, p. 014311, 2014.

[22] W. Dominik et al. "Optical time projection chamber for imaging of twoproton decay of ${ }^{45} \mathrm{Fe}$ nucleus," IEEE Trans. Nucl. Sci., vol. 52, no. 6, pp. 2895-2899, Dec. 2005.

[23] T. Fujiwara, Y. Mitsuya, and H. Toyokawa, "Fine-pitch glass GEM for high-resolution X-ray imaging," J. Instrum., vol. 11, no. 12, p. C12050, 2016.

[24] M. J. French et al., "Design and results from the APV25, a deep submicron CMOS front-end chip for the CMS tracker," Nucl. Instrum. Methods Phys. Res. A, Accel. Spectrom. Detect. Assoc. Equip., vol. 466, no. 2, pp. 359-365, 2001.

[25] S. Martoiu, H. Muller, A. Tarazona, and J. Toledo, "Development of the scalable readout system for micro-pattern gas detectors and other applications," J. Instrum., vol. 8, no. 3, p. C03015, 2013. 